\title{
Reduced Diffusion in Neurocysticercosis: Circumstances of Appearance and Possible Natural History Implications
}

\author{
G.T. Santos, C.C. Leite, L.R. Machado, A.M. McKinney, and L.T. Lucato
}

\begin{abstract}
BACKGROUND AND PURPOSE: Few studies discuss DWI findings in patients with NCC, and their conclusions are variable and contradictory. The aim of our study was to describe DWI findings of a cohort of patients with NCC, emphasizing the frequency of reduced diffusion.

MATERIALS AND METHODS: This retrospective study included 48 patients with NCC. Two neuroradiologists analyzed MR images regarding location, number, and stage of NCC lesions. On the basis of visual analysis, they defined, by consensus, the presence of high signal within NCC lesions on DWI and measured their ADC values when feasible.

RESULTS: The total number of lesions was 342: parenchymal (263), subarachnoid (65), and intraventricular (14); 83 were DWI hyperintense. The first pattern was a small eccentric hyperintense dot/curvilinear structure on DWI (representing the scolex) noted in intraparenchymal lesions in vesicular (41 lesions, 29\%) and colloidal vesicular (18 lesions, 19\%) stages, in 14 (22\%) subarachnoid lesions, and 2 (14\%) intraventricular lesions; rADC calculations were hampered by the intrinsic small dimensions of this finding. The second pattern was the presence of total/subtotal DWI hyperintensity in intraparenchymal lesions, 5 in the colloidal vesicular stage (5\%) and 1 in the granular nodular phase (3\%). Two subarachnoid lesions also showed the same presentation; in this second pattern, reduced diffusion was present in different degrees, measured by rADC calculations.
\end{abstract}

CONCLUSIONS: DWI may identify the scolex, increasing diagnostic confidence for NCC. Total/subtotal DWI hyperintensity, related to the stage of the lesion, though uncommon, allows including NCC as a consideration in the differential diagnosis of lesions with reduced diffusion and ring enhancement.

ABBREVIATIONS: $\mathrm{NCC}=$ neurocysticercosis; $\mathrm{rADC}=$ relative $\mathrm{ADC}$

$\mathbf{N}$ CC, the central nervous system form of cysticercosis, caused by larvae of the tapeworm Taenia solium, is commonly associated with seizures, headache, and focal neurologic deficits. It may also lead to the development of long-term impairment, particularly from epilepsy or hydrocephalus. ${ }^{1-3}$

Neuroimaging techniques, including CT and MR imaging, have revolutionized the diagnosis and management of this disease, by providing evidence of the number and topography of lesions, their stage of evolution, and the degree of inflammatory reaction of the host against the parasites. ${ }^{4-6}$ While some imaging findings are highly suggestive of NCC, in some cases it may be

Received February 22, 2012; accepted after revision April 16.

From the Departments of Radiology (G.T.S., C.C.L., L.T.L.) and Neurology (L.R.M.), Clinics Hospital of the University of Sao Paulo, School of Medicine, Sao Paulo, Brazil; and Department of Radiology/Neuroradiology (A.M.M.), University of Minnesota and Hennepin County Medical Centers, Minneapolis, Minnesota.

Please address correspondence to Leandro Tavares Lucato, MD, Ave Dr. Enéas de Carvalho Aguiar, 255-3 andar, Sao Paulo, Brazil, CEP: 05403-001, e-mail: Itlucato@uol.com.br

http://dx.doi.org/10.3174/ajnr.A3198 challenging to differentiate NCC from other infectious or neoplastic diseases. ${ }^{3-5}$ In this regard, advanced MR imaging techniques have been used in an attempt to increase overall accuracy. ${ }^{7-9}$

DWI has been extensively used in various settings besides its primary clinical use in acute stroke. For example, DWI may be implemented in differentiating brain abscess and tumor ${ }^{10,11}$ or in differentiating epidermoid and arachnoid cysts. ${ }^{12,13}$ Currently, there are few studies discussing the DWI findings in patients with NCC, but the limited studies demonstrate results that are variable and somewhat contradictory: Some studies disclosed hypointense signal within the cysts on DWI, similar to CSF, ${ }^{7,14,15}$ whereas others have depicted the presence of various degrees of reduced diffusion. ${ }^{16,17}$ However, the occurrence of reduced diffusion in patients with NCC has not been entirely understood, particularly regarding the location and relation to the stage of NCC.

The aim of this study was to describe the DWI findings of a cohort of patients with NCC, emphasizing specifically the frequency and the stage of NCC lesions in which DWI abnormalities can be appreciated. 


\section{MATERIALS AND METHODS}

This was a retrospective study, which included patients whose examinations were archived in our teaching file data base. Inclusion criteria were essentially the presence of the examination in the data base (including all relevant sequences necessary for adequate staging of lesions-especially FLAIR, T2*, T1 postgadolinium, and also DWI) and a clinical diagnosis of NCC, confirmed by stringent criteria as detailed elsewhere. ${ }^{18}$ Exclusion criteria were patients whose examinations disclosed only calcified lesions, patients presenting innumerable lesions that were too extensive to count $(>100)$, and incomplete examinations (not all relevant sequences were available).

MR imaging examinations were performed in $1.5 \mathrm{~T}$ or 3T scanners ( 2 patients). Imaging protocol included the following sequences: axial T1-weighted spin-echo, axial T2*, axial and coronal T2-weighted fast spin-echo, and axial FLAIR. Section thickness was $5 \mathrm{~mm}$, and the FOV varied between 18 and $24 \mathrm{~cm}$. After the administration of a gadolinium chelate $(0.1 \mathrm{mmol} / \mathrm{kg})$, an axial 3D T1 sequence (spoiled gradient-recalled echo sequence, section thickness of $1.6 \mathrm{~mm}$ ) and 5-mm-thick T1-weighted spinecho sequences were also obtained.

Before contrast administration, diffusion was studied by obtaining axial echo-planar images (TR/TE in 1.5T and 3T, 10,000/105.3 and $3231 / 67 \mathrm{~ms}$, respectively) with a matrix resolution of $96 \times 128$ $(1.5 \mathrm{~T}) / 192 \times 153(3 \mathrm{~T})$ and with 2 different b-values, 0 and 1000 $\mathrm{s} / \mathrm{mm}^{2}$, corresponding to T2 and DWI, respectively. Section thickness was $5 \mathrm{~mm}$ at $1.5 \mathrm{~T}$ and $4 \mathrm{~mm}$ at $3 \mathrm{~T}$. Diffusion gradients were applied in 3 different directions ( $\mathrm{x}, \mathrm{y}$, and $\mathrm{z}$ ), and ADC maps were generated by calculating $\mathrm{ADC}$ values pixel by pixel. $^{19}$

MR images were analyzed in consensus by 2 neuroradiologists (with 10 and 4 years of experience reading brain MR imaging examinations) regarding the location and number of NCC lesions; the stage of all parenchymal lesions was defined on the basis of previously defined criteria. ${ }^{5,18,20}$ Due to intrinsic difficulties in counting subarachnoid lesions in the racemose form, these were arbitrarily grouped as 1 lesion when detected.

Lesions in the nodular calcified stage were excluded from analysis because MR imaging has well-known limitations in characterizing these (compared with CT). Besides, these lesions are mineralized, punctate, and entirely $\mathrm{T} 2$ hypointense, making ADC measurement challenging and more prone to inaccuracy. ${ }^{21}$

In addition, on the basis of visual analysis of DWI, the presence or absence of high signal in NCC lesions was carefully evaluated in consensus. When DWI hyperintensity was judged present and when the size of the DWI hyperintense portion of the lesion allowed location of a region of interest, the region of interest was manually outlined twice on ADC maps $\left(0.10-0.30 \mathrm{~cm}^{2}\right)$, encompassing the DWI hyperintense portion of a given lesion, and the obtained values were averaged. ADC values were also obtained from corresponding regions of interest outlining contralateral normal-appearing white matter. Calculation of rADC was defined by means of the following formula: ADC of the Lesion/ADC of Contralateral Normal-Appearing White Matter.

\section{RESULTS}

We identified 75 patients from our data base with NCC, 27 of whom were excluded due to the above-cited criteria. Our final

\begin{tabular}{|c|c|c|c|}
\hline \multirow[b]{2}{*}{ Location } & \multirow[b]{2}{*}{$\begin{array}{l}\text { No. of } \\
\text { Lesions }\end{array}$} & \multicolumn{2}{|c|}{ DWI-Hyperintense Lesions } \\
\hline & & $\begin{array}{l}\text { Eccentric (Punctate } \\
\text { or Comma-Shaped) }\end{array}$ & $\begin{array}{c}\text { Total/ } \\
\text { Subtota }\end{array}$ \\
\hline \multicolumn{4}{|l|}{ Intraparenchymal } \\
\hline Vesicular & 139 & 41 & 0 \\
\hline Colloidal vesicular & 93 & 18 & 5 \\
\hline Granular nodular & 31 & 0 & 1 \\
\hline Subarachnoid & 65 & 14 & 2 \\
\hline Intraventricular & 14 & 2 & 0 \\
\hline Total & 342 & 75 & 8 \\
\hline
\end{tabular}

Table 2: NCC lesions presenting total/subtotal DWI hyperintensity

\begin{tabular}{llccc}
\hline Patient & $\begin{array}{c}\text { Lesion Stage/ } \\
\text { Location }\end{array}$ & $\begin{array}{c}\text { ADC-Lesion } \\
\left(\times 10^{-3} \mathbf{m m}^{2} / \mathrm{s}\right)\end{array}$ & $\begin{array}{c}\text { ADC-WM } \\
\left(\times 10^{-3} \mathbf{~ m m}^{2} / \mathrm{s}\right)\end{array}$ & rADC \\
\hline A & Colloidal & $0.94 \pm 0.25$ & $0.96 \pm 0.19$ & 0.98 \\
B & Colloidal & $1.41 \pm 0.38$ & $1.47 \pm 0.13$ & 0.95 \\
C (1) & Colloidal & $0.72 \pm 0.11$ & $0.84 \pm 0.12$ & 0.85 \\
C (2) & Colloidal & $0.52 \pm 0.13$ & $0.75 \pm 0.12$ & 0.68 \\
D & Colloidal & $0.54 \pm 0.16$ & $0.75 \pm 0.12$ & 0.72 \\
E & Granular nodular & $0.51 \pm 0.24$ & $0.94 \pm 0.28$ & 0.54 \\
F & Subarachnoid & $0.80 \pm 0.38$ & $1.01 \pm 0.13$ & 0.79 \\
& (racemose) & & & \\
G & Subarachnoid & $0.68 \pm 0.83$ & $1.18 \pm 0.15$ & 0.57 \\
\hline
\end{tabular}

sample then included 48 patients (30 men and 18 women). Their ages ranged from 17 to 69 years (median, 38 years).

Regarding lesion location, on a per-patient basis, 13 cases presented as a solitary lesion. The remaining 35 patients presented with multiple lesions, ranging from 2 to 35 (mean of 8 lesions per patient).

The total number of lesions inspected was 342, distributed according to their location in parenchymal (263), intraventricular (14), and subarachnoid spaces (65). Twelve of 65 subarachnoid lesions were characterized as having a racemose form. Staging of the 263 parenchymal lesions resulted in the following: 139 lesions in the vesicular stage, 93 lesions in the colloidal vesicular stage, and 31 in the granular nodular phase (Table 1). From the 33 patients with parenchymal lesions, 21 (63.63\%) had coexistence of lesions in different stages, the most common association being between vesicular and colloidal vesicular stages.

Overall, we noticed 83 lesions with DWI hyperintensity (Table 1). In 75 , there was visible evidence of a small eccentric dot or comma-shaped hyperintensity on DWI that might represent the scolex, especially when correlated with other sequences; the small dimensions of this finding, however, precluded a reliable and reproducible measurement of the ADC. Eight lesions (7 patients, classified from A to G in Table 2) presented total/subtotal DWI hyperintensity, associated with the presence of reduced diffusion in different degrees, as measured by rADC calculations.

All cystic lesions judged to be in the vesicular stage showed high signal on T2WI and low signal on T1WI and FLAIR, paralleling CSF signal on all sequences. There was no evidence of relevant contrast enhancement or perilesional edema. DWI demonstrated low signal in all lesions, like CSF. However, in 41 of 139 lesions in the vesicular stage (29.49\%), there was visible evidence of the small eccentric dot or comma-shaped DWI hyperintensity 

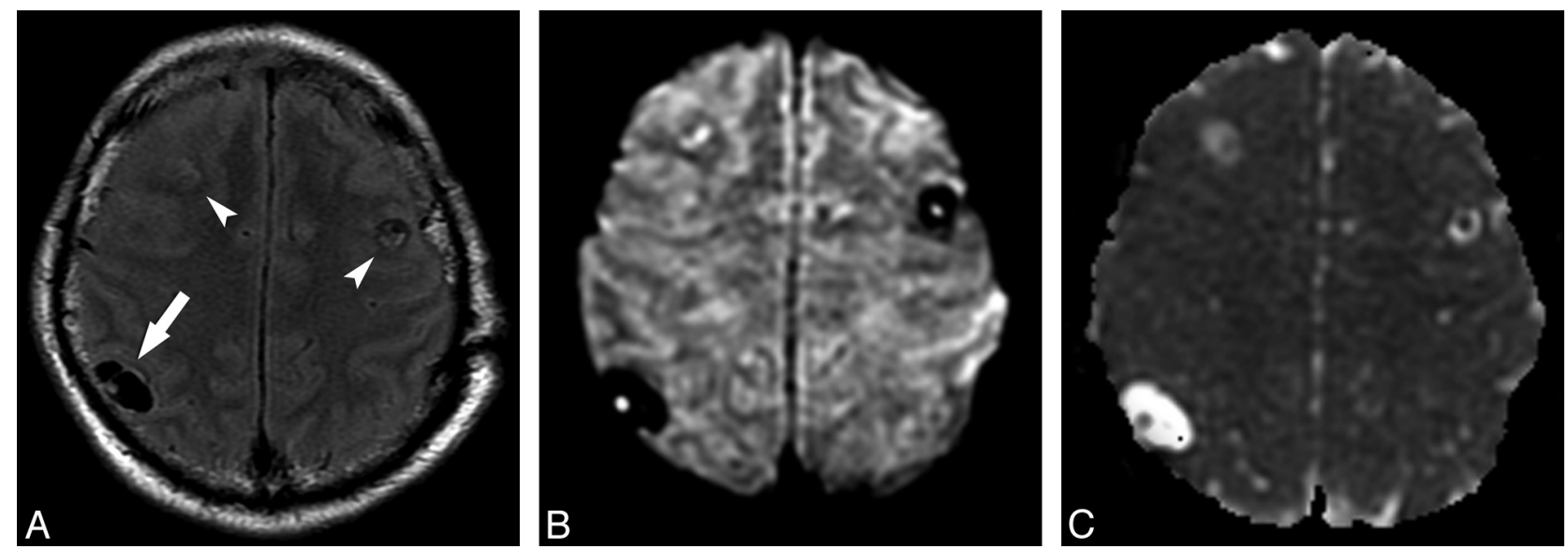

FIG 1. Axial FLAIR $(A)$ and axial DWI $(B)$ images show 3 lesions in both frontoparietal areas (arrow and arrowheads in $A$ ); there is 1 right parietal lesion (arrow on $A$ ), compatible with NCC in the vesicular state. In this lesion, the scolex is clearly seen on DWI as a hyperintense eccentric dot, but it can also be appreciated in the other 2 images. ADC map (C) discloses the scolices as iso-/hypointense dots.
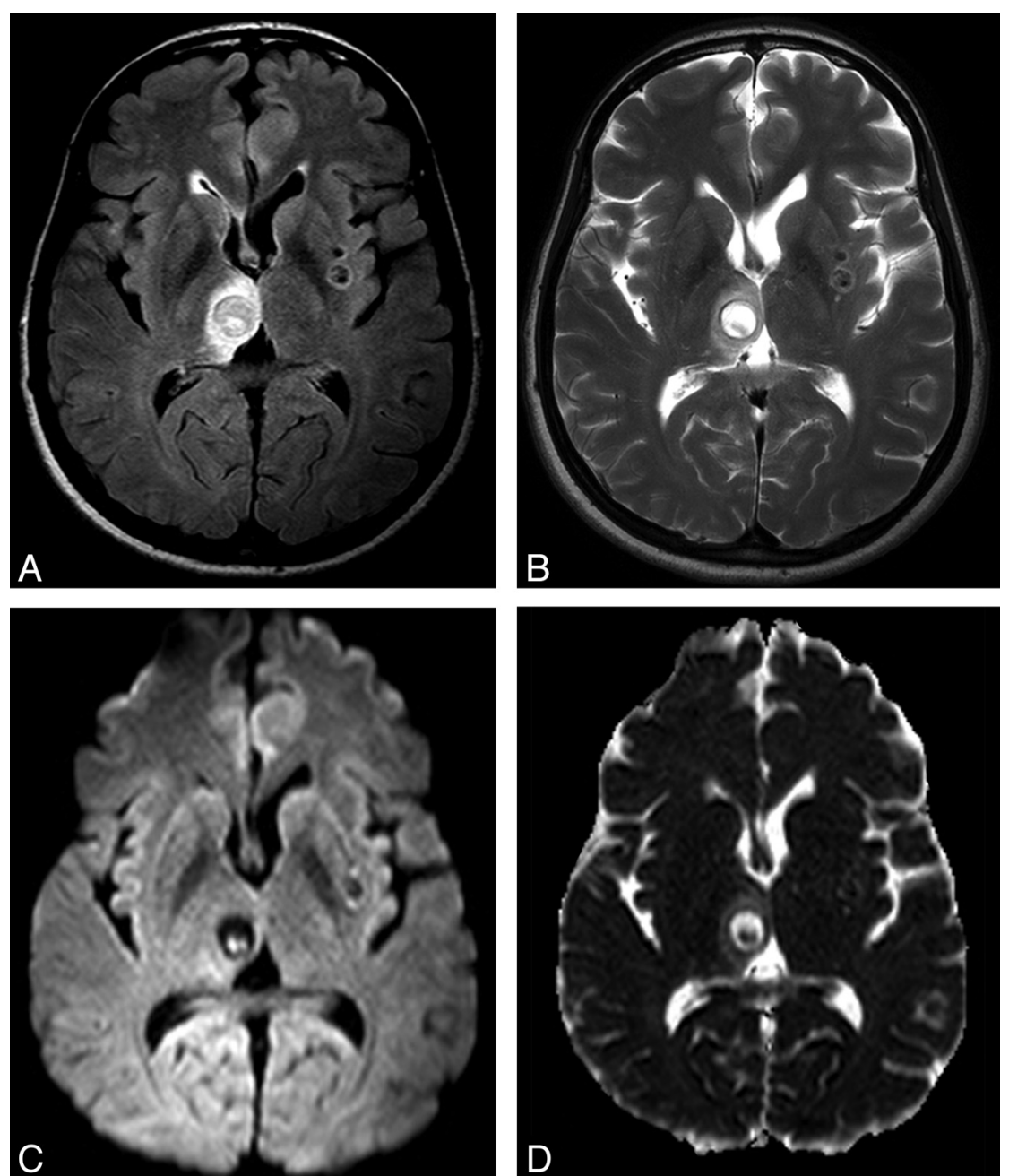

FIG 2. Axial FLAIR $(A)$ and axial T2 fast spin-echo $(B)$ images show lesions in the right thalamus and the left lentiform nucleus; the thalamic lesion is compatible with NCC in the colloidal stage. In this lesion, the curvilinear scolex is clearly seen in a transverse section, on DWI (C), as 2 hyperintense contiguous dots. ADC map $(D)$ discloses the scolex as iso-/hypointense dots.

suggestive of the scolex, which presented iso-/hypointensity in ADC maps compared with brain parenchyma (Fig 1).
In the 93 lesions deemed to be in the colloidal vesicular stage, all showed low signal on T1WI, high signal on T2WI, and intermediate signal on FLAIR. Ringlike enhancement was identified in each of the lesions after contrast administration, with surrounding vasogenic edema. On DWI, 18 of 93 lesions $(19.35 \%)$ presented with the small hyperintensity suggestive of the scolex and iso-/hypointensity in ADC maps (Fig 2); however, 5 (5.37\%) also had total/subtotal DWI hyperintensity without a clearly discernible image, suggestive of the scolex on DWI and conventional sequences (Table 2).

Lesions at the granular nodular stage had low signal on T1WI, low or intermediate signal on T2WI, and intermediate signal on FLAIR images. Each of these lesions demonstrated ringlike enhancement after contrast administration and almost no surrounding vasogenic edema compared with the colloidal vesicular stage lesions. One of 31 lesions (3.22\%) had DWI hyperintensity involving the whole lesion, with low signal on the corresponding ADC maps, confirming the presence of reduced diffusion (Fig 3 and Table 2).

Fourteen of 65 lesions in the subarachnoid space $(21.53 \%)$ had the small DWI hyperintensity suggestive of the scolex and iso-/hypointensity on ADC maps (Fig 4). In 2 of these subarachnoid lesions (16.66\%), complete DWI hyperintensity was detected with low signal on the corresponding ADC maps, confirming the presence of reduced diffusion (Fig 5 and Table 2). Two of 14 intraventricular lesions (14.28\%) also showed the DWI hyperin- 

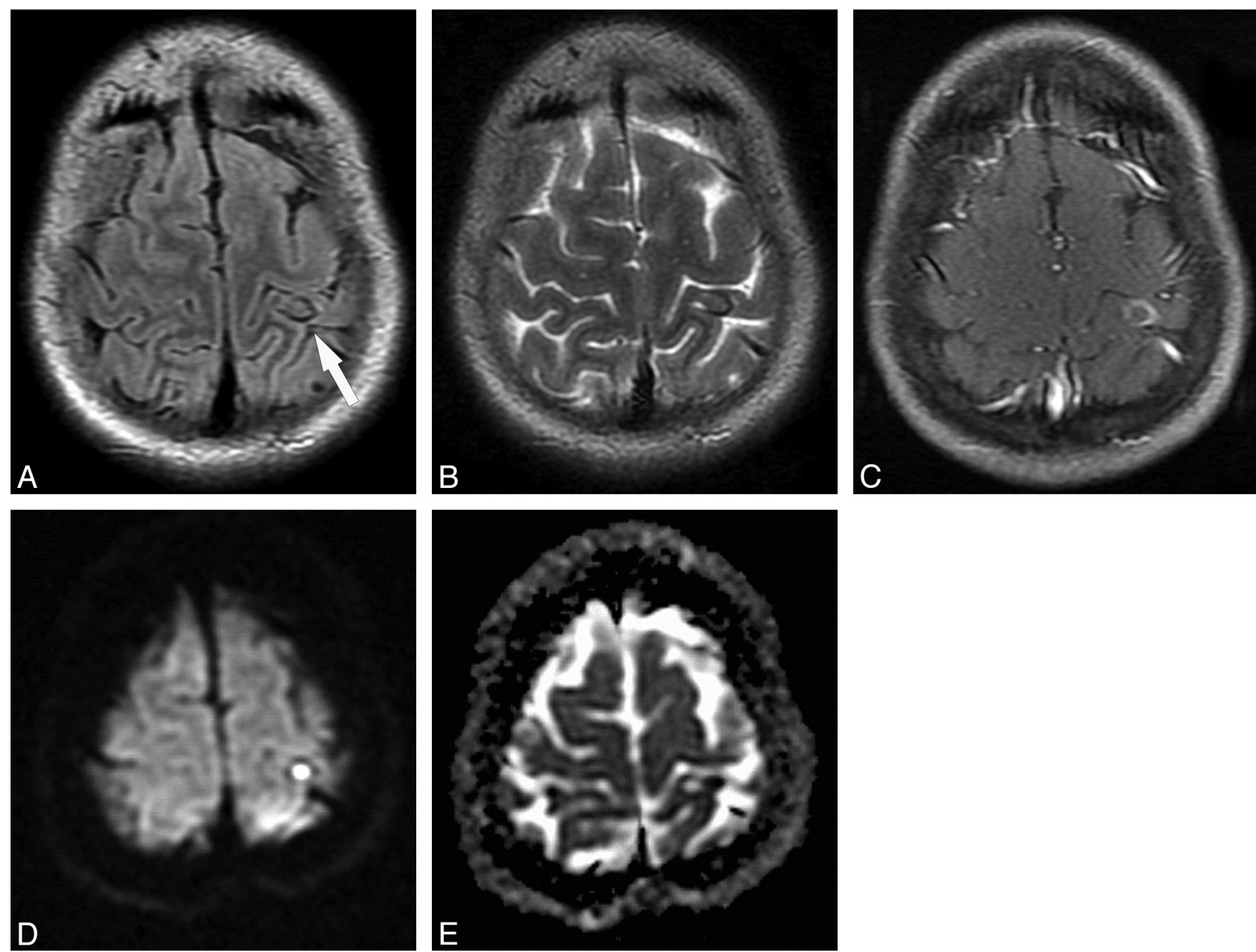

FIG 3. NCC lesion in the granular nodular stage (patient $E$ ) is appreciated in axial FLAIR $(A)$ and T2-weighted (B) images in the left postcentral gyrus (arrow in $A$ ), presenting proteinaceous content (distinct from CSF) with a peripheral rim of low signal presumably due to the beginning of the calcification process, and no perilesional edema. Corresponding axial spin-echo postcontrast T1-weighted $(C)$ image demonstrates residual postcontrast enhancement. Axial DWI image $(D)$ and the corresponding ADC map $(E)$ show homogeneous reduced diffusion of the internal portion of this lesion, possibly related to a more viscous inflammatory content ( $\mathrm{rADC}=0.54$ ).
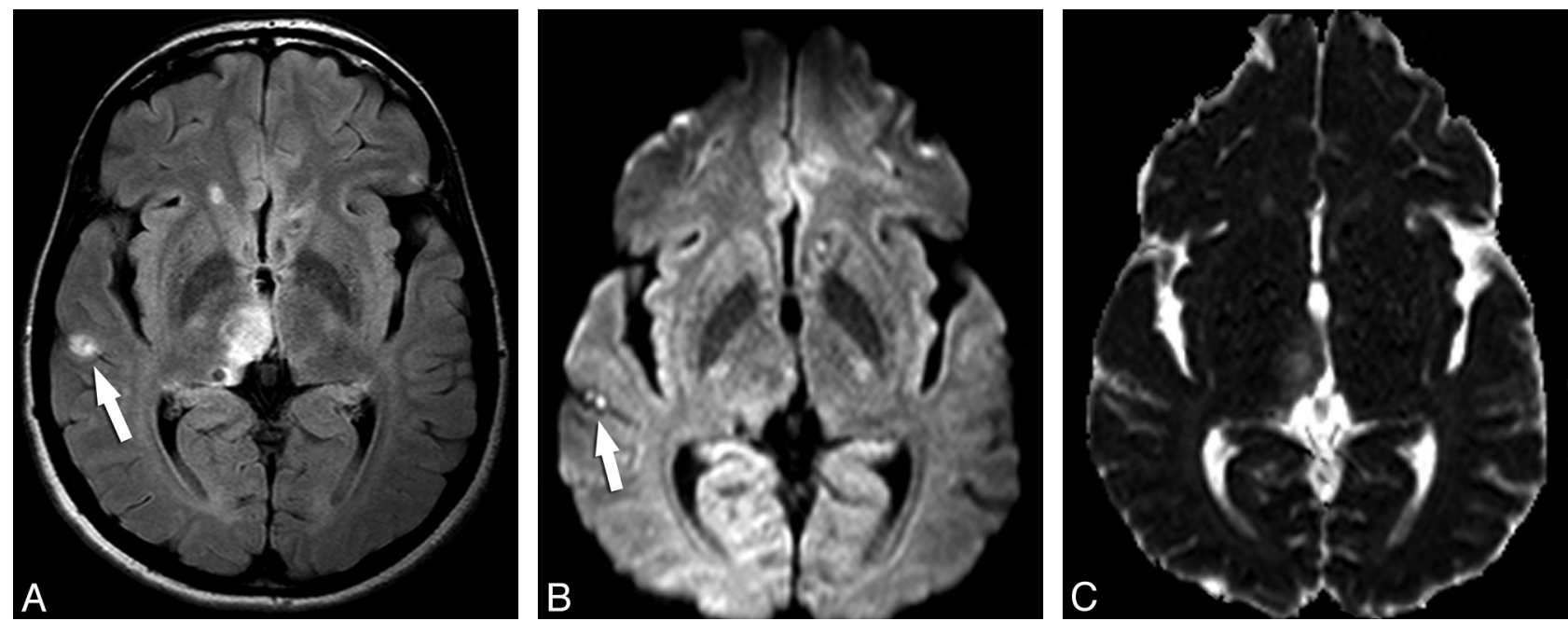

FIG 4. A small subarachnoid NCC lesion whose scolex is depicted only in DWI. Axial FLAIR $(A)$ image demonstrates a hyperintense lesion in the right lateral temporal region (arrow). The DWI image $(B)$ shows the curvilinear scolex in a transverse section as 2 hyperintense contiguous dots (arrow). Other lesions can be appreciated in the right thalamus. ADC map (C) discloses the scolex as iso-/hypointense dots.

tensity presumably corresponding to the scolex and iso-/hypointensity on ADC maps (Fig 6).
Most interesting, though in a minority of cases, the eccentric dot or comma-shaped DWI hyperintensity allowed a more pre- 

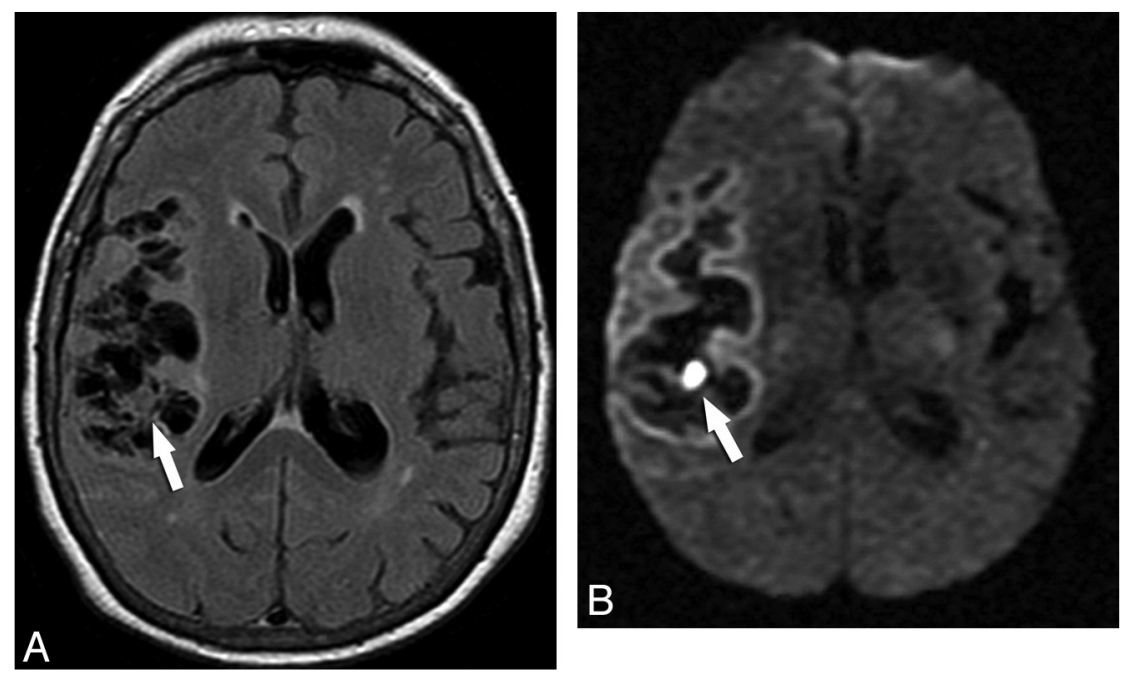

FIG 5. Axial FLAIR sequence $(A)$ in patient $F$ demonstrates many subarachnoid cystic lesions in the right Sylvian cistern, compatible with the racemose form of NCC. Note specifically 1 lesion (arrows) that has signal intensity distinct from CSF. Axial DWI (B) image demonstrates homogeneous reduced diffusion in this small lesion $(\mathrm{rADC}=0.79)$.
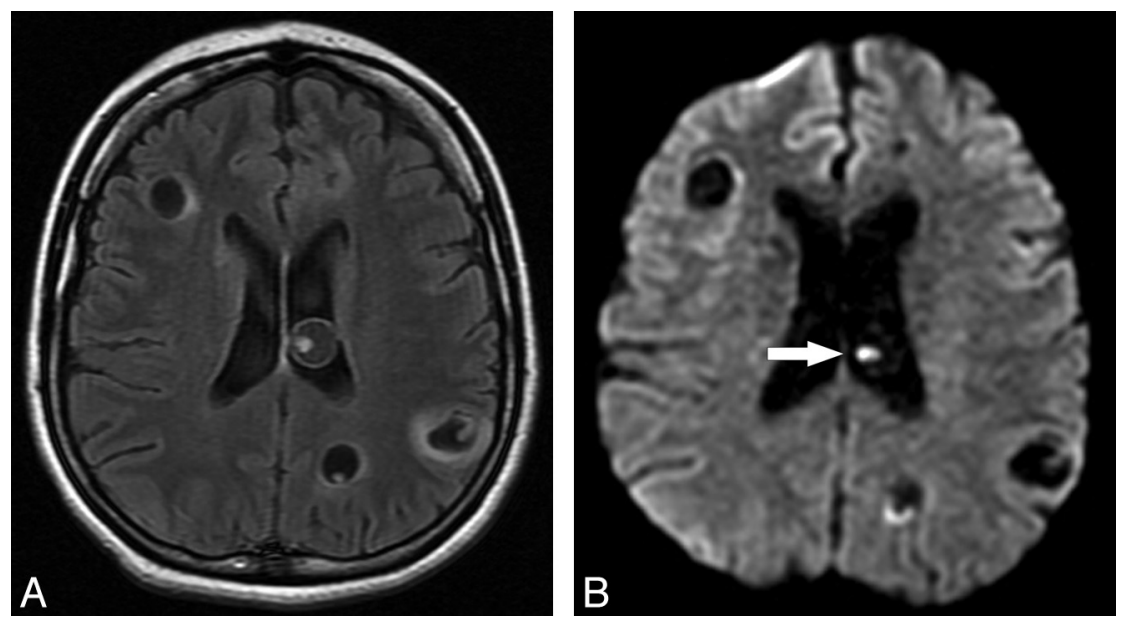

FIG 6. The intraventricular DWI hyperintense scolex is appreciated inside a lesion in the left lateral ventricle. The lesion and its scolex are well seen on the corresponding FLAIR image $(A)$, but the scolex (arrow) is identified as a hyperintense dot in the DWI sequence (B). Additionally, other intraparenchymal NCC lesions are identified bilaterally.

cise identification of the scolex compared with other sequences, including FLAIR. This phenomenon was noticed in 21 lesions in the vesicular stage (15.10\%), 13 lesions in the colloidal vesicular stage (13.97\%), and 7 (10.76\%) in the subarachnoid space (Fig 4$)$.

\section{DISCUSSION}

NCC is considered the most common parasitic disease affecting the central nervous system, and besides the well-known endemic locations, it has rising importance in developed countries. ${ }^{22,23}$ Adding complexity to the scenario, NCC is clinically a pleomorphic disease. ${ }^{24}$ The imaging findings of NCC are also quite variable, and the coexistence of lesions in different stages and locations is frequently observed, ${ }^{4,6}$ in agreement with the current study. Some lesions present a diagnostic challenge regarding their location, particularly in distinguishing parenchymal and subarachnoid compartments. ${ }^{25}$ Our study criteria designated lesions as subarachnoid only when unequivocally belonging to this location; otherwise these were counted as parenchymal.

The MR imaging evolution of parenchymal NCC lesions recognizes 4 stages: vesicular, colloidal vesicular, granular nodular, and nodular calcified. In the vesicular stage, the cysticercus is viable, there is no inflammation, and it has the characteristics of a CSF-like signal cyst presenting with the scolex as an eccentric mural nodule. When the lesion evolves to the colloidal vesicular stage, degeneration commences and inflammation plays a central role, manifest as perilesional edema and peripheral contrast enhancement; the signal intensity of the lesion at that time is distinct from CSF (hyperintense on FLAIR) as the scolex starts to degenerate. The parasite is already dead by the granular nodular stage as the retracted lesion begins to mineralize (showing T2hypointensity), and the edema and enhancement progressively subside. In the nodular calcified stage, the lesion achieves complete mineralization. ${ }^{6,26}$ The fact that calcification is the hallmark of the final evolutionary stage of the cysticercus is another point that has led us to exclude patients presenting with only this kind of lesion, because DWI would not be expected to add much value at that point.

Our sample demonstrated an eccentric DWI hyperintense dot or curvilinear structure in a subset of NCC lesions during both the vesicular and colloidal vesicular stages. The assumption that it represents the scolex comes from correlating with other sequences on which the scolex can be discerned. Previous work from Raffin et $a{ }^{17}$ described this finding in 7 of 11 patients with NCC, but there was no mention of lesion count as a whole or per patient. Lesions in this study were also not classified according to the disease stage, though the authors stated that all patients had cystic lesions with signal intensity similar to that of CSF, with or without peripheral edema and/or enhancement, ${ }^{17}$ which signals that their sample probably included lesions in the vesicular stage as well as in transitional forms between vesicular and colloidal vesicular stages, because even the lesions with edema or enhancement exhibited CSF signal in that study.

We have expanded their observation to a larger sample population, classifying lesions according to NCC stages, including the depiction of a DWI hyperintense eccentric structure in lesions belonging to the colloidal vesicular stage, whose content was clearly distinct from CSF on FLAIR images. The mechanism underlying DWI-hyperintense scolices is so far unknown: maybe these represent the earliest sign of cyst degeneration (which can be 
ascertained by future, prospective studies); otherwise, differences related to the host immune response or genetic variations of the parasite might explain this finding. ${ }^{27,28}$

The eccentric DWI hyperintensity presumably representing the scolex has an important diagnostic implication because the detection of the scolex is pathognomonic of NCC and considered an absolute diagnostic criterion of the disease. ${ }^{18}$ Due to its small dimensions, reliable measurements of rADC could not be obtained, but the qualitative finding of iso-/hypointensity on ADC maps indicates that the T2 shine through effect is less likely. The depiction of this sign was clearly visible on DWI in a subset of patients and thus likely has direct clinical applicability, increasing diagnostic confidence even when conventional MR images already point to a diagnosis of NCC, because DWI is already incorporated in MR imaging protocols in daily clinical practice.

We also demonstrated that reduced diffusion inside a lesion can occur in parenchymal lesions in either the colloidal vesicular or granular nodular stages, and reduced diffusion may also occur within the subarachnoid space. In this setting, reduced diffusion possibly is a hallmark of a viscous and/or proteinaceous content, which is expected during degeneration of a cystic NCC lesion. Using diffusion tensor imaging, Gupta et $\mathrm{al}^{29}$ recently found, in 25 patients, a progressive decrease in mean diffusivity and an increase in fractional anisotropy during evolution of NCC lesions from vesicular to colloidal vesicular and from colloidal vesicular to granular nodular stages, which corroborates our observation. One can speculate that lesions in the colloidal vesicular stage with reduced diffusion represent a "late" colloidal vesicular stage, in contrast to an "early" colloidal vesicular stage in which the signal intensity of the lesion still resembles CSF. If one applies the same concept, lesions in the granular nodular stage with reduced diffusion might represent an "early" granular nodular stage; when calcification becomes relevant, in the so-called "late" granular nodular stage, reduced diffusion disappears as the lesion contents evolve from a viscous liquid to a solid material, signaling transition to the last stage of development of NCC lesions, the nodular calcified phase. In short, it seems that the DWI appearance in NCC lesions may relate to a continuum of degeneration, characterized by a tendency to reduced diffusion that ceases when a solid state is achieved ("late" granular nodular stage).

When scrutinizing the pertinent literature, we found early works investigating the value of DWI in the differential diagnosis of cystic brain lesions. Park et $\mathrm{al}^{16}$ examined 39 cystic or necrotic intracranial lesions, including 12 cases of NCC; all lesions showed signal intensity similar to or slightly higher than that of CSF on T1- and T2-weighted images (they did not use FLAIR sequences). The authors observed variable signal intensity in patients with cysticercosis, including 3 parenchymal lesions in the colloidal vesicular stage and 1 cisternal lesion presenting with high signal on DWI. Because FLAIR images were not obtained (the most reliable when one needs to compare the signal intensity of a lesion with the CSF), we can only speculate about the content of these lesions relative to CSF. Most likely, the lesions in that study that had reduced diffusion would present with frank hyperintensity on FLAIR images, related to what we refer to in this study as the "late" colloidal vesicular stage.

Bergui et $\mathrm{al}^{14}$ studied the DWI findings in 24 patients with cystic brain lesions, including 1 case of ring-enhancing NCC. This single patient exhibited no signs of reduced diffusion on the images provided in that study, though again FLAIR images were not provided. We surmise that this appearance could represent an "early" colloidal vesicular stage lesion. A similar analysis and conclusion could be formed from the study of Mishra et al, ${ }^{7}$ who studied 3 cystic ring-enhancing NCC lesions, and also from Reddy et $\mathrm{al},{ }^{30}$ who analyzed 2 similar NCC lesions.

Finally, Gupta et $\mathrm{al}^{15}$ intended to differentiate intracranial tuberculoma and tuberculous abscess from cysticercal granulomas on the basis of DWI. They found no reduced diffusion in 16 NCC lesions in the vesicular stage and in 29 lesions in the colloidal vesicular and granular nodular stages, but FLAIR images were again not obtained. In this study, they collected fluid from some lesions at the time of surgery, and though the protein contents were not significantly different between degenerating NCC and tuberculous lesions, the cellularity and viscosity were significantly distinct (NCC lesions were devoid of cells and less viscous). This could, at least in part, account for the absence of reduced diffusion seen on these NCC lesions.

Our data support the inclusion of NCC as a possibility in the differential diagnosis of cystic lesions with reduced diffusion and ring enhancement, together with bacterial abscesses, ${ }^{11}$ fungal infection, ${ }^{31}$ and tuberculomas/tuberculous abscesses. ${ }^{15}$ Therefore, another aspect reinforcing the clinical relevance of the present work is that in some cases, it was easier to characterize the lesion and the scolex on DWI than on other sequences. Hence, the findings described in this study on DWI could be useful to help distinguish NCC from other lesions in the differential diagnosis.

Limitations of this study include the following: lack of pathologic and CT correlations, a retrospective and transverse design, and patients not consecutively included (due to characteristics of the above-cited inclusion criteria). However, patients usually presenting with $>1$ lesion and with various stages of evolution makes the sample collected here quite relevant. Prospective longitudinal studies with pathologic correlation are encouraged to address some of the issues presented here and to advance the knowledge of such an important disease.

\section{CONCLUSIONS}

DWI findings in NCC may relate to the stage of infection. In vesicular and colloidal vesicular stages, the scolex can present as a DWI hyperintense dot or comma-shaped structure. Total/subtotal DWI hyperintensity may be present in cystic parenchymal lesions in the colloidal vesicular and granular nodular phases. Thus, DWI may signal a continuum of degeneration.

This constellation of findings has direct and relevant clinical implications. DWI may be important in increasing diagnostic confidence for NCC. NCC, albeit rarely, can also be considered in the differential diagnosis of cystic lesions with reduced diffusion, ring enhancement, and vasogenic edema; such a list also includes fungal and bacterial abscesses.

Disclosures: Claudia Leite-UNRELATED: Payment for Lectures (including service on Speakers Bureaus): I was invited and paid to participate in a Guerbet meeting, Other: The institution did a clinical trial with Guerbet last year. Alexander McKinneyUNRELATED: Board Membership: Vital Images Medical Advisory Board member, Comments: less than $\$ 5000 /$ year monetary compensation; research equipment usage.

AJNR Am J Neuroradiol 34:310-16 Feb 2013 www.ajnr.org 315 


\section{REFERENCES}

1. Carpio A. Neurocysticercosis: an update. Lancet Infect Dis 2002;2:751-62

2. Hawk MW, Shahlaie K, Kim KD, et al. Neurocysticercosis: a review. Surg Neurol 2005;63:123-32

3. Narata AP, Arruda WO, Uemura E, et al. Neurocysticercosis: a tomographic diagnosis in neurological patients [in Portuguese]. Arq Neuropsiquiatr 1998;56:245-49

4. Garcia $\mathrm{HH}$, Del Brutto $\mathrm{OH}$. Imaging findings in neurocysticercosis. Acta Trop 2003;87:71-78

5. Noujaim SE, Rossi MD, Rao SK, et al. CT and MR imaging of neurocysticercosis. AJR Am J Roentgenol 1999;173:1485-90

6. Lucato LT, Guedes MS, Sato JR, et al. The role of conventional MR imaging sequences in the evaluation of neurocysticercosis: impact on characterization of the scolex and lesion burden. AJNR Am J Neuroradiol 2007;28:1501-04

7. Mishra AM, Gupta RK, Jaggi RS, et al. Role of diffusion-weighted imaging and in vivo proton magnetic resonance spectroscopy in the differential diagnosis of ring-enhancing intracranial cystic mass lesions. J Comput Assist Tomogr 2004;28:540-47

8. Mont'Alverne Filho FE, Machado L dos R, Lucato LT, et al. The role of $3 \mathrm{D}$ volumetric $\mathrm{MR}$ sequences in diagnosing intraventricular neurocysticercosis: preliminary results. Arq Neuropsiquiatr 2011;69:74-78

9. do Amaral LL, Ferreira RM, da Rocha AJ, et al. Neurocysticercosis: evaluation with advanced magnetic resonance techniques and atypical forms. Top Magn Reson Imaging 2005;16:127-44

10. Kim YJ, Chang KH, Song IC, et al. Brain abscess and necrotic or cystic brain tumor: discrimination with signal intensity on diffusion-weighted MR imaging. AJR Am J Roentgenol 1998;171:1487-90

11. Ebisu T, Tanaka C, Umeda M, et al. Discrimination of brain abscess from necrotic or cystic tumors by diffusion-weighted echo planar imaging. Magn Reson Imaging 1996;14:1113-16

12. Hakyemez B, Aksoy U, Yildiz H, et al. Intracranial epidermoid cysts: diffusion-weighted, FLAIR and conventional MR findings. Eur J Radiol 2005;54:214-20

13. Tsuruda JS, Chew WM, Moseley ME, et al. Diffusion-weighted MR imaging of the brain: value of differentiating between extraaxial cysts and epidermoid tumors. AJNR Am J Neuroradiol 1990;11:925-31

14. Bergui M, Zhong J, Bradac GB, et al. Diffusion-weighted images of intracranial cyst-like lesions. Neuroradiology 2001;43:824-29

15. Gupta RK, Prakash M, Mishra AM, et al. Role of diffusion weighted imaging in differentiation of intracranial tuberculoma and tuberculous abscess from cysticercus granulomas-a report of more than 100 lesions. Eur J Radiol 2005;55:384-92

16. Park SH, Chang KH, Song IC, et al. Diffusion-weighted MRI in cystic or necrotic intracranial lesions. Neuroradiology 2000;42:716-21

17. Raffin LS, Bacheschi LA, Machado LR, et al. Diffusion-weighted MR imaging of cystic lesions of neurocysticercosis: a preliminary study. Arq Neuropsiquiatr 2001;59:839-42

18. Del Brutto OH, Rajshekhar V, White AC Jr, et al. Proposed diagnostic criteria for neurocysticercosis. Neurology 2001;57:177-83

19. Schaefer PW, Grant PE, Gonzalez RG. Diffusion-weighted MR imaging of the brain. Radiology 2000;217:331-45

20. Amaral L, Maschietto M, Maschietto R, et al. Unusual manifestations of neurocysticercosis in MR imaging: analysis of 172 cases. Arq Neuropsiquiatr 2003;61:533-41

21. Maldjian JA, Listerud J, Moonis G, et al. Computing diffusion rates in T2-dark hematomas and areas of low T2 signal. AJNR Am J Neuroradiol 2001;22:112-18

22. Sinha S, Sharma BS. Neurocysticercosis: a review of current status and management. J Clin Neurosci 2009;16:867-76

23. Kraft R. Cysticercosis: an emerging parasitic disease. Am Fam Physician 2007;76:91-96

24. Carabin H, Ndimubanzi PC, Budke CM, et al. Clinical manifestations associated with neurocysticercosis: a systematic review. PLoS Negl Trop Dis 2011;5:e1152

25. Kimura-Hayama ET, Higuera JA, Corona-Cedillo R, et al Neurocysticercosis: radiologic-pathologic correlation. Radiographics 2010;30:1705-19

26. Abdel Razek AA, Watcharakorn A, Castillo M. Parasitic diseases of the central nervous system. Neuroimaging Clin $N \mathrm{Am}$ 2011;21:815-41

27. Campbell G, Garcia HH, Nakao M, et al. Genetic variation in Taenia solium. Parasitol Int 2006;55(suppl):S121-26

28. Pittella JE. Neurocysticercosis. Brain Pathol 1997;7:681-93

29. Gupta RK, Trivedi R, Awasthi R, et al. Understanding changes in DTI metrics in patients with different stages of neurocysticercosis. Magn Reson Imaging 2012;30:104-11

30. Reddy JS, Mishra AM, Behari S, et al. The role of diffusion-weighted imaging in the differential diagnosis of intracranial cystic mass lesions: a report of 147 lesions. Surg Neurol 2006;66:246-50

31. Gaviani P, Schwartz RB, Hedley-Whyte ET, et al. Diffusion-weighted imaging of fungal cerebral infection. AJNR Am J Neuroradiol 2005; 26:1115-21 